\title{
Decentralized Supply Chain Performance When Demand Information Is Not Shared
}

\author{
Youssef Tliche $^{1, *}$, Atour Taghipour ${ }^{2}$, Béatrice Canel-Depitre ${ }^{3}$ \\ ${ }^{1}$ University of Le Havre Normandie, France \\ ${ }^{2}$ University of Le Havre Normandie, France \\ ${ }^{3}$ University of Le Havre Normandie, France \\ *Corresponding author: youssef.tliche @ univ-lehavre.fr
}

\begin{abstract}
In decentralized logistic structures, information management policies generally do not allow information sharing among actors of the same supply chain, or in the best cases, allow the sharing of some non-key information. In order to enhance overall decentralized supply chain performance, we investigate a recent strategy called "Downstream Demand Inference" which allows an upstream actor to infer the demand at his formal downstream actor without the need of formal information sharing. Downstream Demand Inference was surveyed through several forecasting methods, and was successfully experimented with only Simple Moving Average method. Thus, in this paper, we investigate Downstream Demand Inference through other method, namely, the Weighted Moving Average forecasting method which affects nonequal weighting to past observations. First, we establish the Mean Squared Error and Average Inventory Level expressions for the upstream actor. Second, we formalize the upstream actor's Forecast Optimization Problem and propose the application of the Newton's method to solve it. Finally, based on simulated time-series demands, the results show that our approach allows further optimized solutions, in terms of forecast errors and inventory levels, which can improve the competitiveness of companies in the market.
\end{abstract}

Keywords: weighted moving average; supply chain management; newton method; downstream demand inference; autoregressive moving average processes

\section{Introduction}

The international economic structure is a complex structure connecting many actors over the world. A producer can be located at the west of the globe when his formal customer, a downstream actor can be located at the east of the globe. Because of the micro-complexity of such structure, sharing all the data between two actors of the same supply chain, or in the best case, sharing the key-data is not a simple task. Most of economic actors generally focus on achieving their own objectives, it's about maximizing their local profits without consideration of partners' objectives. Thus, a self-serving focus often leads to poor overall performance. However, an enhanced overall performance is achievable if the companies of the same supply chain coordinate their operations such that each company's performance participate to overall the supply chain performance. 
Supply Chain Management (SCM) is one of the most important research area that aims to improve overall supply chains performance. SCM refers to the set of operations potentially able to common performance of a supply chain. In this context, information sharing is presented as an effective approach to ameliorate supply chain performance (Cachon \& Fisher, 2000; Yu et al., 2001; Sahin \& Robinson, 2005). Unfortunately, there are many practical limitations for information sharing (Lee \& Whang, 2000; Mendelson, 2000; Fawcett et al., 2007; Forslund \& Jonsson, 2007; Klein et al., 2007). It's first about confidential policies. Generally, companies don not allow sharing key-information because they estimate that such data are monetaryvaluable. Second, it's also about data reliability. Many companies do not rely on shared data and estimate that errors are so high to be seriously considerable. Third, even in the rare cases when actors are favorite for coordination, intra-organization systems can resist against changes. Many companies consider that intra-organizational costs are so high to implement coordination systems. In addition, the incompatibility of information systems between "geographically and culturally" distant actors of the same supply chain is another type of information sharing barriers.

In the midst of this research area, a new coordination supply chain approach, known as Downstream Demand Inference (DDI) (Ali et al., 2017; Ali \& Boylan, 2011; Ali \& Boylan, 2012, Tliche et al., 2019), appeared thus allowing the enhancement of a decentralized supply chain without having to go through explicit key-information sharing. Instead of sharing final customer's demand information arriving at a downstream actor, the upstream actor is able to infer the demand at his formal downstream actor, to be used in the forecasts. A substitute hypothesis for information sharing is that DDI coordination approach assume that customer's demand process and its parameters are known over the supply chain. It was also shown (Ali \& Boylan, 2011; Ali \& Boylan, 2012) that DDI is not possible with Minimum Mean Squared Error (MMSE) method or Single Exponential Smoothing (SES) because the propagation of demand over the supply chain may not be unique, but possible when the downstream actor uses the Simple Moving Average (SMA) forecasting method which considers equal weights to past observations. For a purpose of investigation, Ali et al. (2017) showed that DDI allows the enhacement of a decentralized supply chain performance in terms of forecast Mean Squared Error (MSE) and average inventory levels/costs under the hypothesis of an $A R(1)$ customer's demand model. Tliche et al. (2019) generalized the above DDI's results for causal invertible $\operatorname{ARMA}(p, q)$ demand models.

In order to produce more-enhanced solutions, we estimated that it's natural to investigate the application of DDI approach through other forecasting methods. In this paper, we propose the consideration of non-equal weights on past observations. Thus, the Weighted Moving Average (WMA) method was considered and an optimization method, namely the Newton method (Qi \& Sun, 1999), was applied in order to determine "optimal" weighting.

The rest of the paper is organizing as follows. Section 2 is devoted to the models and methodology. Section 3 is devoted to the simulation results. Finally, in Section 4, we carry some discussions on our results and natural perspectives. 


\section{Models and Methodology}

\subsection{Supply chain model}

We first consider a simple two-level decentralized supply chain formed by an upstream actor and a downstream actor who receives the demand of a final customer. We suppose that a periodic review system is adopted for replenishment, where the downstream actor place his order at the upstream actor after examining his respective inventory level. Indeed, after the realization of the demand $D_{t}$ at the downstream actor at the beginning of time period $t$, and after checking his own inventory level, he places an order of size $Y_{t}$ before the end of the period. Then, the upstream actor prepares the required order $Y_{t}$ and ships it to the downstream actor, who will receive it at period $t+L+1$. The parameter $L$ refers to the replenishment time of both production and shipment.

On one hand, it's assumed that there is no order cost. On the other hand, unit inventory holding cost and shortage cost are constant and denoted respectively by $h$ and $s$. It's also assumed that both upstream actor and downstream actor adopt Order-Up-To (OUT) policy which minimizes the total costs over infinite time horizon (Lee et al., 2000).

\subsubsection{Customer's demand model and WMA forecasting method}

We assume that customer's demand arriving at the downstream actor is a causal invertible ${ }^{1}$ $\operatorname{ARMA}(p, q)$ process. Let $D_{t}$ be the demand process at the downstream actor, such as Eq. 1:

$$
D_{t}=c+\sum_{j=1}^{p} \phi_{j} D_{t-j}+\xi_{t}+\sum_{j=1}^{q} \theta_{j} \xi_{t-j}
$$

where $c \geq 0$ is the unconditional mean of the demand process, $\phi_{j}, j \in\{1, \ldots, p\}$ are the autoregressive coefficients of the demand process, $\theta_{j}, j \in\{1, \ldots, q\}$ are the moving average coefficients of the demand process and $\xi_{t} \sim N\left(0, \sigma_{\xi}^{2}\right), t \in[0,+\infty$ [ are the independently and identically distributed error terms, according to the normal distribution.

Furthermore, let $\gamma_{k}=\operatorname{Cov}\left(d_{t+k}, d_{t}\right)$ be the covariance between demands at periods $t$ and $t+k$. This definition is required for some formulas derivations in this work.

As mentioned, instead of considering SMA method, we propose to use WMA method which, at period $(t+1)$, can be mathematically written by Eq. 2:

$$
f_{t+1}=\sum_{i=1}^{N} x_{i} D_{t+1-i}
$$

\footnotetext{
${ }^{1}$ Please refer to Shumway \& Stoffer (2011) for more details on ARMA models, causality and invertibility.
} 
We define $x=\left(\begin{array}{c}x_{1} \\ : \\ x_{N}\end{array}\right)$ the weighting vector where $x_{i}$ is the ponderation/weight associated to customer's demand at time period $t+1-i$, and verifying the set of constraints $(C):\left\{\begin{array}{rl} & \sum_{i=1}^{N} x_{i}=1 \\ x_{i} \geq 0 & 0 i \in\{1, \ldots, N\}\end{array}\right.$

In order to apply DDI strategy, it's firstly important to check whether the propagation of the demand across the supply chain is unique.

\subsubsection{Downstream actor's order time-series structure}

Let $Y_{t}$ be the order process at the upstream actor, such as Eq. 3:

$$
Y_{t}=c+\sum_{j=1}^{p} \phi_{j} Y_{t-j}+\tilde{\xi}_{t}+\sum_{j=1}^{q} \theta_{j} \tilde{\xi}_{t-j}
$$

where $c \geq 0$ is the unconditional mean of the order process, $\phi_{j}, j \in\{1, \ldots, p\}$ are the autoregressive coefficients of the order process, $\theta_{j}, j \in\{1, \ldots, q\}$ are the moving average coefficients of the order process and $\xi_{t} \sim N\left(0,\left(2 L^{2} \sum_{i=1}^{N} x_{i}^{2}+2 L x_{1}+1\right) \sigma_{\xi}^{2}\right), t \in[0,+\infty[$ are the independently and identically distributed error terms, according to the normal distribution.

We note that the demand and order processes have the same autoregressive and moving average coefficients, and differ only by their respective error terms. Indeed, the order's error terms are amplificated by a coefficient $\beta=2 L^{2} \sum_{i=1}^{N} x_{i}^{2}+2 L x_{1}+1$ such as $\sigma_{\tilde{\xi}}^{2}=\beta \sigma_{\xi}^{2}$. Consequently, the order process is unique and the upstream actor is able to infer the demand process without need of demand information sharing.

\section{Proof:}

Using WMA method, we can define the lead-time demand forecast $f_{t+1}^{L}$ which is given by Eq. 3a as follows:

$$
f_{t+1}^{L}=L\left(\sum_{i=1}^{N} x_{i} D_{t+1-i}\right)
$$

Now, the OUT level is calculated by:

$$
S_{t}=f_{t+1}^{L}+z \Sigma
$$

Where $z$ is the safety factor and $\Sigma$ is the standard deviation of the noise in the lead-time demand (Silver et al., 1998). The orders from the downstream actor to the upstream actor are calculated by summing the demand at the downstream actor plus any change in the OUT level in the current period, and given by Eq. 3c and Eq. $3 \mathrm{~d}$.

$$
\begin{gathered}
Y_{t}=S_{t}-S_{t-1}+D_{t} \\
\Leftrightarrow Y_{t}=f_{t+1}^{L}+z \Sigma-\left(f_{t}^{L}+z \Sigma\right)+D_{t}
\end{gathered}
$$




\section{International Conference on}

\section{MANAGEMENT, ECONOMICS \& FINANCE}

$$
\Leftrightarrow Y_{t}=f_{t+1}^{L}-f_{t}^{L}+D_{t}
$$

Substituing $f_{t+1}^{L}$ ad $f_{t}^{L}$ by their respective expressions in Eq. 3d, we obtain the following results:

$$
\begin{gathered}
Y_{t}=L\left(x_{1} D_{t}-x_{1} D_{t-1}+x_{2} D_{t-1}-x_{2} D_{t-2}+\cdots+x_{N} D_{t-N+1}-x_{N} D_{t-N}\right)+D_{t} \\
\Leftrightarrow Y_{t}=c+\phi_{1} Y_{t-1}+\cdots+\phi_{p} Y_{t-p}+\left[L\left(\sum_{i=1}^{N} x_{i}\left(\xi_{t-i+1}-\xi_{t-i}\right)\right)+\xi_{t}\right] \\
+\theta_{1}\left[L\left(\sum_{i=1}^{N} x_{i}\left(\xi_{t-i}-\xi_{t-i-1}\right)\right)+\xi_{t-1}\right] \\
+\theta_{2}\left[L\left(\sum_{i=1}^{N} x_{i}\left(\xi_{t-i-1}-\xi_{t-i-2}\right)\right)+\xi_{t-2}\right]+\cdots \\
+\theta_{q}\left[L\left(\sum_{i=1}^{N} x_{i}\left(\xi_{t-i-q+1}-\xi_{t-i-q}\right)\right)+\xi_{t-q}\right]
\end{gathered}
$$

Let consider $\tilde{\xi}_{t}=L\left(\sum_{i=1}^{N} x_{i}\left(\xi_{t-i+1}-\xi_{t-i}\right)\right)+\xi_{t}$, so $Y_{t}$ can finally be expressed by Eq. $3 \mathrm{e}$ as follows:

$$
Y_{t}=c+\phi_{1} Y_{t-1}+\cdots+\phi_{p} Y_{t-p}+\tilde{\xi}_{t}+\theta_{1} \tilde{\xi}_{t-1}+\theta_{2} \tilde{\xi}_{t-2}+\cdots+\theta_{q} \tilde{\xi}_{t-q}
$$

and finally, we obtain Eq. 3 .

Hence, $Y_{t}$ follows an $\operatorname{ARMA}(p, q)$ process where $\tilde{\xi}_{t} \sim N\left(0,\left(2 L^{2} \sum_{i=1}^{N} x_{i}^{2}+2 L x_{1}+1\right) \sigma_{\xi}^{2}\right)$ are the error terms. Indeed, it's easy to check the following errors properties given by Eq. $3 \mathrm{f}$ and $3 \mathrm{~g}$ as follows:

$$
E\left(\tilde{\xi}_{t}\right)=E\left(L\left(\sum_{i=1}^{N} x_{i}\left(\xi_{t-i+1}-\xi_{t-i}\right)\right)+\xi_{t}\right)=0
$$

and

$$
\begin{gathered}
\operatorname{Var}\left(\tilde{\xi}_{t}\right)=\operatorname{Var}\left(L\left(\sum_{i=1}^{N} x_{i}\left(\xi_{t-i+1}-\xi_{t-i}\right)\right)+\xi_{t}\right) \\
\Leftrightarrow \operatorname{Var}\left(\tilde{\xi}_{t}\right)=\left(2 L^{2} \sum_{i=1}^{N} x_{i}^{2}+2 L x_{1}+1\right) \sigma_{\xi}^{2}
\end{gathered}
$$

As all parameters of $Y_{t}: c, \phi_{j}$ and $\theta_{j}$ are unique, the order process $Y_{t}$ arriving at the upstream actor is also unique and thus, the upstream actor is able to infer the demand arriving at his formal downstream actor.

Next, we consider the two upstream performance metrics, namely the upstream forecast mean squared error and average inventory level, as they present the engine of the whole supply 


\section{International Conference on}

\section{MANAGEMENT, ECONOMICS \& FINANCE}

chain performance. Specifically, these cost savings generated by an inventory holdings decrease are the basis benefits on which the upstream actor can propose a benefit sharing contract to his formal downstream actor.

\subsection{Supply chain methodology}

\subsubsection{Upstream actor's forecast mean squared error and average inventory levels}

Under DDI strategy, the mean squared error is expressed by Eq. 4a as follows:

$$
\begin{aligned}
& M S E^{D D I}=\operatorname{Var}\left[\sum_{i=1}^{L+1}\left(D_{t+i}-f_{t+i}\right)\right]=\operatorname{Var}\left[\sum_{i=1}^{L+1} D_{t+i}-(L+1) f_{t+1}\right] \\
& \Leftrightarrow M S E^{D D I}=\operatorname{Var}\left(\sum_{i=1}^{L+1} D_{t+i}\right)+(L+1)^{2} \operatorname{Var}\left(f_{t+1}\right) \\
&-2(L+1) \operatorname{Cov}\left(\sum_{i=1}^{L+1} D_{t+i}, f_{t+1}\right)
\end{aligned}
$$

We then derive the three components of Eq. 4a. The first term is given by Eq. $4 \mathrm{~b}$ (Tliche et al., 2018) as follows:

$$
\operatorname{Var}\left(\sum_{i=1}^{L+1} D_{t+i}\right)=(L+1) \gamma_{0}+2 \sum_{i=1}^{L} i \gamma_{L+1-i}
$$

The second term of Eq. 4a can be expressed by Eq. 4c as follows, after some mathematical operations:

$$
\begin{gathered}
\operatorname{Var}\left(f_{t+1}\right)=\operatorname{Var}\left(\sum_{i=1}^{N} x_{i} D_{t+1-i}\right) \\
\Leftrightarrow \operatorname{Var}\left(f_{t+1}\right)=\gamma_{0} \sum_{i=1}^{N} x_{i}{ }^{2}+2 \sum_{j=1}^{N-1}\left(x_{j} \sum_{i=j+1}^{N} x_{i} \gamma_{i-j}\right) \\
\Leftrightarrow(L+1)^{2} \operatorname{Var}\left(f_{t+1}\right)=(L+1)^{2}\left[\gamma_{0} \sum_{i=1}^{N} x_{i}{ }^{2}+2 \sum_{j=1}^{N-1}\left(x_{j} \sum_{i=j+1}^{N} x_{i} \gamma_{i-j}\right)\right]
\end{gathered}
$$

And lastly, the third term of Eq. $4 \mathrm{a}$ can be expressed by Eq. $4 \mathrm{~d}$ as follows, after some mathematical manipulations:

$$
\operatorname{Cov}\left(\sum_{i=1}^{L+1} D_{t+i}, f_{t+1}\right)==\sum_{i=1}^{L+1} \sum_{j=1}^{N} x_{j} \gamma_{i+j-1}
$$




\section{International Conference on}

\section{MANAGEMENT, ECONOMICS \& FINANCE}

$$
\Leftrightarrow-2(L+1) \operatorname{Cov}\left(\sum_{i=1}^{L+1} D_{t+i}, f_{t+1}\right)=-2(L+1) \sum_{i=1}^{L+1} \sum_{j=1}^{N} x_{j} \gamma_{i+j-1}
$$

So finally, we obtain the mean squared error expression given by Eq. 4 as follows:

$$
\begin{aligned}
& M S E^{D D I}(x)=(L+1) \gamma_{0}+2 \sum_{i=1}^{L} i \gamma_{L+1-i} \\
& +(L+1)^{2}\left[\gamma_{0} \sum_{i=1}^{N} x_{i}^{2}+2 \sum_{j=1}^{N-1}\left(x_{j} \sum_{i=j+1}^{N} x_{i} \gamma_{i-j}\right)\right] \\
& -2(L+1) \sum_{i=1}^{L+1} \sum_{j=1}^{N} x_{j} \gamma_{i+j-1}
\end{aligned}
$$

Next, the average inventory level under OUT policy is given by Eq. 5a (Ali et al., 2012) as follows:

$$
\tilde{I}_{t}=T_{t}-E\left(\sum_{i=1}^{L+1} Y_{t+i}\right)+\frac{E\left(Y_{t}\right)}{2}
$$

where the upstream actor's optimal OUT inventory level $T_{t}$ is expressed by $T_{t}=M_{t}+K \sigma_{\tilde{\xi}} \sqrt{V}$ and $M_{t}$ and $V$ are respectively, the conditional expectation and conditional variance of the total demand over the lead-time, and $K=F_{N(0,1)}^{-1}\left(\frac{s}{s+h}\right)$ for the standard normal distribution $F_{N(0,1)}$.

Under DDI strategy and using WMA forecast method, the average inventory level is expressed by Eq. $5 \mathrm{~b}$ as follows:

$$
\begin{gathered}
\tilde{I}_{t}^{D D I}(x)=T_{t}^{D D I}(x)-E\left(\sum_{i=1}^{L+1} Y_{t+i}\right)+\frac{E\left(Y_{t}\right)}{2} \\
\Leftrightarrow \tilde{I}_{t}^{D D I}(x)=M_{t}^{D D I}(x)+K \sigma_{\tilde{\xi}} \sqrt{V^{D D I}(x)}-E\left(\sum_{i=1}^{L+1} Y_{t+i}\right)+\frac{E\left(Y_{t}\right)}{2}
\end{gathered}
$$

Through some mathematical manipulations, we can demonstrate that:

- $M_{t}^{D D I}(x)=\frac{c(L+1)}{\left(1-\sum_{j=1}^{p} \phi_{j}\right)}$

- $V^{D D I}(x)=M S E^{D D I}(x)$

- $-E\left(\sum_{i=1}^{L+1} Y_{t+i}\right)=-c(L+1)\left(1-\sum_{j=1}^{p} \phi_{j}\right)^{-1}$

- $E\left(Y_{t}\right)=c\left(1-\sum_{j=1}^{p} \phi_{j}\right)^{-1}$ 


\section{MANAGEMENT, ECONOMICS \& FINANCE}

By substituing all the above terms by their respective expressions in Eq. 5b, we finally obtain Eq. $5 \mathrm{c}$ and Eq. 5 as follows:

$$
\begin{gathered}
\tilde{I}_{t}^{D D I}(x)=\frac{c}{2\left(1-\sum_{j=1}^{p} \phi_{j}\right)}+K \sigma_{\tilde{\xi}} \sqrt{M S E^{D D I}(x)} \\
\tilde{I}_{t}^{D D I}(x)=\frac{c}{2\left(1-\sum_{j=1}^{p} \phi_{j}\right)} \\
+K \sigma_{\tilde{\xi}}\left[(L+1) \gamma_{0}+2 \sum_{i=1}^{L} i \gamma_{L+1-i}\right. \\
+(L+1)^{2}\left[\gamma_{0} \sum_{i=1}^{N} x_{i}{ }^{2}+2 \sum_{j=1}^{N-1}\left(x_{j} \sum_{i=j+1}^{N} x_{i} \gamma_{i-j}\right)\right] \\
\left.-2(L+1) \sum_{i=1}^{L+1} \sum_{j=1}^{N} x_{j} \gamma_{i+j-1}\right]^{\frac{1}{2}}
\end{gathered}
$$

We note that the average inventory level expression in Eq. $5 \mathrm{c}$ is a non-linear function of the forecast mean squared error, which can explain the non-proportional evolution linking these two performance metrics.

\subsubsection{Newton method for "optimal" weighting}

We assume that the upstream actor aims to minimize his average inventory levels $\tilde{I}_{t}^{D D I}(x)$ while forecasting over the time period $L+1$. As mentioned before, This inventory-oriented enhancement will be the main key of the whole supply chain performance. To do so, the upstream actor can simply find out the minimal mean squared error and then substitute its expression in Eq. 5 by its value. This minimal value is determined by implementing the wellknown Newton optimization method which allows the minimization of quadratic convex functions.

First, let us mathematically formulate this problem, namely the forecast optimization problem, denoted by $\mathrm{P}$ as follows:

$$
(\mathrm{P}):\left\{\begin{array}{c}
\text { minimize } \\
\operatorname{MSE}^{D D I}(x) \\
\text { subject to }\left\{\begin{array}{c}
\sum_{i=1}^{N} x_{i}=1 \\
x_{i} \geq 0 \forall i=1, \ldots, N \\
x=\left(\begin{array}{c}
x_{1} \\
\vdots \\
x_{N}
\end{array}\right)
\end{array}\right.
\end{array}\right.
$$

The problem $\mathrm{P}$ is reformulated into matricial form and becomes as follows: 


\section{International Conference on}

\section{MANAGEMENT, ECONOMICS \& FINANCE}

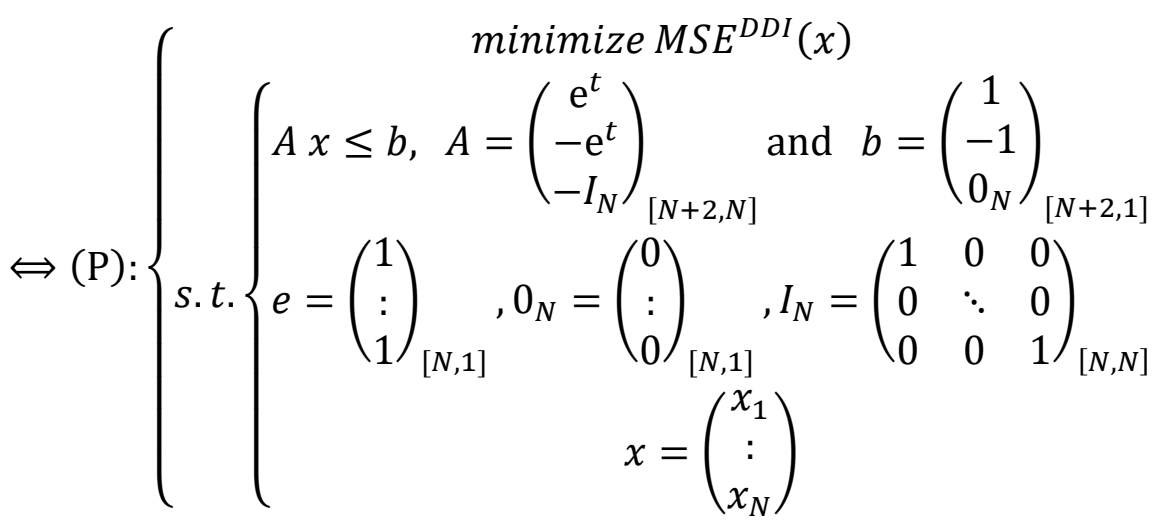

Next, we define the necessary Karush-Kuhn-Tucker (K.K.T.) (Gordon \& Tibshirani, 2012) first order optimality conditions associated with this problem and thus we obtain $\mathrm{P}^{\prime}$.

$$
\left(\mathrm{P}^{\prime}\right):\left\{\begin{array}{c}
\text { (1): } \quad \frac{\partial M S E^{D D I}(x)}{\partial x}+A^{t} \lambda=0_{N} \\
\text { (2): } \lambda_{i}(A x-b)_{i}=0, \quad i=1, \ldots, r \\
A x \leq b \\
\text { s.t. }\left\{\begin{array}{c}
\lambda_{i} \geq 0, \quad i=1, \ldots, r \\
r=N+2 \\
x=\left(\begin{array}{c}
x_{1} \\
: \\
x_{N}
\end{array}\right) \\
\lambda=\left(\begin{array}{c}
\lambda_{1} \\
: \\
\lambda_{r}
\end{array}\right)
\end{array}\right.
\end{array}\right.
$$

The derivative function of the mean squared error can be expressed by Eq. 6 as follows:

$$
\left.\frac{\partial M S E^{D D I}(x)}{\partial x}=\left[\begin{array}{c}
2(L+1)^{2}\left(\gamma_{0} x_{1}+\sum_{\substack{i=1 \\
i \neq 1}}^{N} x_{i} \gamma_{|i-1|}\right)-2(L+1) \sum_{i=1}^{L+1} \gamma_{i} \\
\vdots \\
2(L+1)^{2}\left(\gamma_{0} x_{N}+\sum_{\substack{i=1 \\
i \neq N}}^{N} x_{i} \gamma_{|i-N|}\right.
\end{array}\right)-2(L+1) \sum_{i=N}^{L+N} \gamma_{i}\right]_{[N, 1]}
$$

We denote $G(x)=\frac{\partial M S E^{D D I}(x)}{\partial x}+A^{t} \lambda$ such as $\mathrm{P}^{\prime}$ becomes:

$$
\left(\mathrm{P}^{\prime}\right):\left\{\begin{array}{c}
G(x, \lambda)=0_{N} \\
\lambda_{i}(b-A x)_{i}=0, \quad \lambda_{i} \geq 0, \quad(b-A x)_{i} \geq 0, \quad i=1, \ldots, r
\end{array}\right.
$$




\section{International Conference on}

\section{MANAGEMENT, ECONOMICS \& FINANCE}

Proposition (Chen et al., 2000) :

If $a \geq 0, b \geq 0, a b=0 \Leftrightarrow \varphi(a, b)=0$ with $\varphi(a, b)=a+b-\sqrt{a^{2}+b^{2}}$

We apply the above proposition to the transformed problem $\mathrm{P}^{\prime}$ and we obtain:

$$
\left(\mathrm{P}^{\prime}\right):\left\{\begin{array}{c}
G(x, \lambda)=0_{N} \\
\psi_{i}\left(\lambda_{i},(b-A x)_{i}\right)=\lambda_{i}+(b-A x)_{i}-\sqrt{\lambda_{i}{ }^{2}+(b-A x)_{i}{ }^{2}}=0, \quad i=1, \ldots, r
\end{array}\right.
$$

Let denote $g\left(\begin{array}{l}x \\ \lambda\end{array}\right)=\left(\begin{array}{l}G(x, \lambda) \\ \psi(x, \lambda)\end{array}\right)=0_{N+r}$

Thus, solving the problem $\mathrm{P}^{\prime}$ is equivalent to solve the following equations system $\mathrm{S}$ :

$$
\text { (S): } \quad\left(\begin{array}{l}
x^{k+1} \\
\lambda^{k+1}
\end{array}\right)=\left(\begin{array}{l}
x^{k} \\
\lambda^{k}
\end{array}\right)-\nabla g^{-1}\left(\begin{array}{l}
x^{k} \\
\lambda^{k}
\end{array}\right) \cdot g\left(\begin{array}{l}
x^{k} \\
\lambda^{k}
\end{array}\right)
$$

The resolution of such equations system requires the computation of the inverse of the hessian matrix $\nabla g^{-1}\left(\begin{array}{l}x^{k} \\ \lambda^{k}\end{array}\right)$ at each iteration $k$, which could be expensive in terms of time and memory. Consequently, the alternative can be the resolution of a linear system with the PivotGauss method (Sorensen, 1985).

Finally, solving S amounts to solving $\mathrm{S}^{\prime}$ :

$$
\left(\mathrm{S}^{\prime}\right): \nabla g\left(\begin{array}{l}
x^{k} \\
\lambda^{k}
\end{array}\right) \cdot \Delta u^{k}=-g\left(\begin{array}{l}
x^{k} \\
\lambda^{k}
\end{array}\right)
$$

where

- $\Delta u^{k}=\left(\begin{array}{l}\Delta x^{k} \\ \Delta \lambda^{k}\end{array}\right)=\left(\begin{array}{l}x^{k+1}-x^{k} \\ \lambda^{k+1}-\lambda^{k}\end{array}\right)$

- $\nabla g\left(\begin{array}{l}x^{k} \\ \lambda^{k}\end{array}\right)=\left[\begin{array}{ll}\frac{\partial G\left(x^{k}, \lambda^{k}\right)}{\partial x} & \frac{\partial G\left(x^{k}, \lambda^{k}\right)}{\partial \lambda} \\ \frac{\partial \psi\left(x^{k}, \lambda^{k}\right)}{\partial x} & \frac{\partial \psi\left(x^{k}, \lambda^{k}\right)}{\partial \lambda}\end{array}\right]_{[N+r, N+r]}$

with

$$
\text { - } \quad \frac{\partial G\left(x^{k}, \lambda^{k}\right)}{\partial x}=\frac{\partial^{2} M S E^{D D I}\left(x^{k}\right)}{\partial x^{2}}=\left[\begin{array}{ccc}
2(L+1)^{2} \gamma_{0} & \cdots & 2(L+1)^{2} \gamma_{|1-N|} \\
\vdots & \ddots & \vdots \\
2(L+1)^{2} \gamma_{|N-1|} & \cdots & 2(L+1)^{2} \gamma_{0}
\end{array}\right]
$$

- $\quad \frac{\partial G\left(x^{k}, \lambda^{k}\right)}{\partial \lambda}=A^{t}$

- $\quad \frac{\partial \psi\left(x^{k}, \lambda^{k}\right)}{\partial x}=-A+\frac{\left(b-A x^{k}\right)_{i} A}{\sqrt{\left(\lambda_{i}^{k}\right)^{2}+\left(b-A x^{k}\right)_{i}^{2}}}$

- $\quad \frac{\partial \psi\left(x^{k}, \lambda^{k}\right)}{\partial \lambda}=\left(1-\frac{\lambda_{i}}{\left(\lambda_{i}^{k}\right)^{2}+\left(b-A x^{k}\right)_{i}^{2}}\right) I_{(r, r)}$ 


\section{Simulation results}

In this section, we carry out some simulated experiments on our implementation, namely the resolution of some examples that will serve for approach validation. Then, we carry some discussions about observed results.

\subsection{Comparative study between SMA and WMA/Newton methods}

In this first part of simulation, we first consider each of demand models in Tab. 1, which are $\operatorname{ARMA}(p, q)$ models, and where we variate autoregressive $\phi_{j}, j \in\{1, \ldots, p\}$ and moving average $\theta_{j}, j \in\{1, \ldots, q\}$ coefficients. Then, for the following fixed parameters: $c=10 ; \sigma_{\xi}^{2}=$ $1 ; L=5 ; N=12 ; h=1 ; s=2$, we make a comparative study between DDI strategy where SMA method is adopted and DDI strategy WMA/Newton methods are adopted. The results obtained for the SMA method are based on the simulation results of Tliche et al. (2019). The arbitrarily chosen parameters of the Newton's algorithm are as follows: $\varepsilon=10^{-5}$ and $i_{\max }=$ 100. The initial solution can be randomly chosen as long as it belongs to the realm of feasible solutions. Newton's algorithm does not exceed a dozen of iterations and the elapsed time is at the scale of the second on a Windows 7 professional system. Tab. 1 and Tab. 2 resume the findings on 20 different simulated demand models.

\begin{tabular}{|c|c|c|}
\hline Demand model & $\begin{array}{l}\text { Autoregressive } \\
\text { and moving } \\
\text { average } \\
\text { coefficients }\end{array}$ & $\begin{array}{c}\text { optimal Newton weighting } \\
x^{*}\end{array}$ \\
\hline 1 & $\phi_{1}=0.400$ & $\left(\begin{array}{l}0.2218 ; 0.0667 ; 0.0667 ; 0.0667 ; 0.0667 ; 0.0667 ; \\
0.0667 ; 0.0667 ; 0.0667 ; 0.0667 ; 0.0667 ; 0.1112\end{array}\right)$ \\
\hline 2 & $\phi_{1}=0.500$ & $\left(\begin{array}{ccc}0.2835 ; 0.0597 ; 0.0597 ; 0.0597 ; 0.0597 ; & 0.0597 ; \\
0.0597 ; 0.0597 ; 0.0597 ; 0.0597 ; 0.0597 ; & 0.1194\end{array}\right)$ \\
\hline 3 & $\phi_{1}=0.600$ & $\left(\begin{array}{ccc}0.3653 ; 0.0508 ; 0.0508 ; 0.0508 ; 0.0508 ; 0.0508 ; \\
0.0508 ; 0.0508 ; 0.0508 ; 0.0508 ; 0.0508 ; 0.1269\end{array}\right)$ \\
\hline 4 & $\theta_{1}=0.400$ & $\left(\begin{array}{c}0.1727 ; 0.0370 ; 0.0913 ; 0.0696 ; 0.0782 ; 0.0749 ; \\
0.0758 ; 0.0764 ; 0.0739 ; 0.0806 ; 0.0636 ; 0.1061\end{array}\right)$ \\
\hline 5 & $\theta_{1}=0.500$ & $\left(\begin{array}{ccc}0.1952 ; 0.0143 ; 0.1046 ; 0.0596 ; 0.0818 ; & 0.0714 ; \\
0.0753 ; 0.0760 ; 0.0704 ; 0.0837 ; 0.0560 ; 0.1118\end{array}\right)$ \\
\hline 6 & $\theta_{1}=0.600$ & $\left(\begin{array}{ccc}0.2116 ; 0.0000 ; 0.1171 ; & 0.0467 ; 0.0880 ; 0.0659 ; \\
0.0756 ; & 0.0756 ; 0.0659 ; 0.0880 ; 0.0476 ; 0.1171\end{array}\right)$ \\
\hline 7 & $\begin{array}{l}\phi_{1}=0.400 \\
\theta_{1}=0.051\end{array}$ & $\left(\begin{array}{ccc}0.2397 ; 0.0567 ; 0.0661 ; 0.0656 ; 0.0656 ; 0.0656 ; \\
0.0656 ; 0.0656 ; & 0.0656 ; 0.0657 ; 0.0631 ; 0.1149\end{array}\right)$ \\
\hline 8 & $\begin{array}{l}\phi_{1}=0.400 \\
\theta_{1}=0.100\end{array}$ & $\left(\begin{array}{ccc}0.2569 ; 0.0455 ; 0.0666 ; 0.0645 ; 0.0647 ; 0.0647 ; \\
0.0647 ; 0.0647 ; 0.0646 ; 0.0652 ; 0.0593 ; 0.1186\end{array}\right)$ \\
\hline
\end{tabular}


International Conference on

MANAGEMENT, ECONOMICS \& FINANCE

15-17 March 2019

\begin{tabular}{|c|c|c|}
\hline 9 & $\begin{array}{l}\phi_{1}=0.400 \\
\theta_{1}=0.300\end{array}$ & $\left(\begin{array}{ccc}0.3186 ; 0.0000 ; 0.0752 ; & 0.0575 ; 0.0628 ; 0.0613 ; \\
0.0615 ; 0.0621 ; 0.0596 ; 0.0680 ; 0.0400 ; & 0.1334\end{array}\right)$ \\
\hline 10 & $\begin{array}{l}\phi_{1}=0.400 \\
\theta_{1}=0.300 \\
\theta_{2}=0.100\end{array}$ & $\left(\begin{array}{ccc}0.3550 ; 0.0000 ; 0.0449 ; 0.0692 ; 0.0574 ; 0.0586 ; \\
0.0593 ; 0.0585 ; 0.0616 ; 0.0565 ; 0.0413 ; 0.1378\end{array}\right)$ \\
\hline 11 & $\begin{array}{l}\phi_{1}=0.400 \\
\theta_{1}=0.300 \\
\theta_{2}=0.150\end{array}$ & $\left(\begin{array}{ccc}0.3733 ; 0.0000 ; 0.0271 ; 0.0758 ; 0.0572 ; 0.0557 ; \\
0.0582 ; 0.0578 ; 0.0624 ; 0.0504 ; 0.0420 ; 0.1400\end{array}\right)$ \\
\hline 12 & $\begin{array}{l}\phi_{1}=0.400 \\
\theta_{1}=0.300 \\
\theta_{2}=0.200\end{array}$ & $\left(\begin{array}{ccc}0.3909 ; 0.0015 ; 0.0068 ; 0.0829 ; 0.0593 ; 0.0514 ; \\
0.0567 ; 0.0583 ; 0.0631 ; 0.0441 ; 0.0428 ; 0.1423\end{array}\right)$ \\
\hline 13 & $\begin{aligned} \phi_{1} & =0.400 \\
\theta_{1} & =0.300 \\
\theta_{2} & =0.180 \\
\theta_{3} & =0.060 \\
\theta_{4} & =0.050\end{aligned}$ & $\left(\begin{array}{ccc}0.4138 ; 0.0091 ; 0.0259 ; 0.0590 ; 0.0410 ; 0.0602 ; \\
0.0562 ; 0.0493 ; 0.0551 ; 0.0464 ; 0.0425 ; 0.1415\end{array}\right)$ \\
\hline 14 & $\begin{array}{l}\phi_{1}=0.200 \\
\phi_{2}=0.150 \\
\theta_{1}=0.100\end{array}$ & $\left(\begin{array}{ccc}0.2194 ; 0.1045 ; 0.0584 ; 0.0630 ; 0.0626 ; 0.0626 ; \\
0.0626 ; 0.0626 ; 0.0627 ; 0.0614 ; 0.0742 ; 0.1059\end{array}\right)$ \\
\hline 15 & $\begin{array}{l}\phi_{1}=0.200 \\
\phi_{2}=0.150 \\
\phi_{3}=0.120 \\
\phi_{4}=0.100 \\
\theta_{1}=0.100\end{array}$ & $\left(\begin{array}{c}0.2806 ; 0.1607 ; 0.1142 ; 0.0694 ; 0.0308 ; 0.0347 ; \\
0.0344 ; 0.0336 ; 0.0415 ; 0.0509 ; 0.0615 ; 0.0878\end{array}\right)$ \\
\hline 16 & $\begin{aligned} \phi_{1} & =0.200 \\
\phi_{2} & =0.150 \\
\phi_{3} & =0.120 \\
\phi_{4} & =0.100 \\
\theta_{1} & =0.100 \\
\theta_{2} & =0.065\end{aligned}$ & $\left(\begin{array}{ccc}0.3018 ; 0.1767 ; 0.0979 ; 0.0623 ; 0.0261 ; 0.0321 ; \\
0.0335 ; 0.0318 ; 0.0389 ; 0.0463 ; 0.0629 ; 0.0899\end{array}\right)$ \\
\hline 17 & $\begin{array}{c}\phi_{1}=0.200 \\
\phi_{2}=0.150 \\
\phi_{3}=0.120 \\
\phi_{4}=0.100 \\
\theta_{1}=0.100 \\
\theta_{2}=0.065 \\
\theta_{3}=0.060 \\
\theta_{4}=0.051\end{array}$ & $\left(\begin{array}{ccc}0.3293 ; 0.1994 ; 0.1171 ; 0.0501 ; 0.0000 ; 0.0177 ; \\
0.0256 ; 0.0257 ; 0.0357 ; 0.0468 ; 0.0629 ; 0.0898\end{array}\right)$ \\
\hline 18 & $\begin{array}{rr}\phi_{1}= & 0.200 \\
\phi_{2}= & -0.150 \\
\phi_{3}= & 0.120 \\
\phi_{4}= & -0.100 \\
\phi_{5}= & 0.080 \\
\phi_{6}= & 0.070 \\
\phi_{7}= & 0.060 \\
\phi_{8}= & -0.051 \\
\theta_{1}= & 0.100\end{array}$ & $\left(\begin{array}{ccc}0.1484 ; 0.0679 ; & 0.1154 ; 0.0721 ; 0.1023 ; 0.0792 ; \\
0.0672 ; 0.0589 ; 0.0657 ; 0.0760 ; 0.0606 ; 0.0865\end{array}\right)$ \\
\hline 19 & $\begin{array}{rr}\phi_{1}= & 0.200 \\
\phi_{2}= & -0.150 \\
\phi_{3}= & 0.120 \\
\phi_{4}= & -0.100\end{array}$ & $\left(\begin{array}{ccc}0.1614 ; 0.0772 ; 0.1089 ; 0.0709 ; 0.0992 ; 0.0770 ; \\
0.0627 ; 0.0550 ; 0.0642 ; 0.0734 ; 0.0617 ; 0.0884\end{array}\right)$ \\
\hline
\end{tabular}


International Conference on

MANAGEMENT, ECONOMICS \& FINANCE

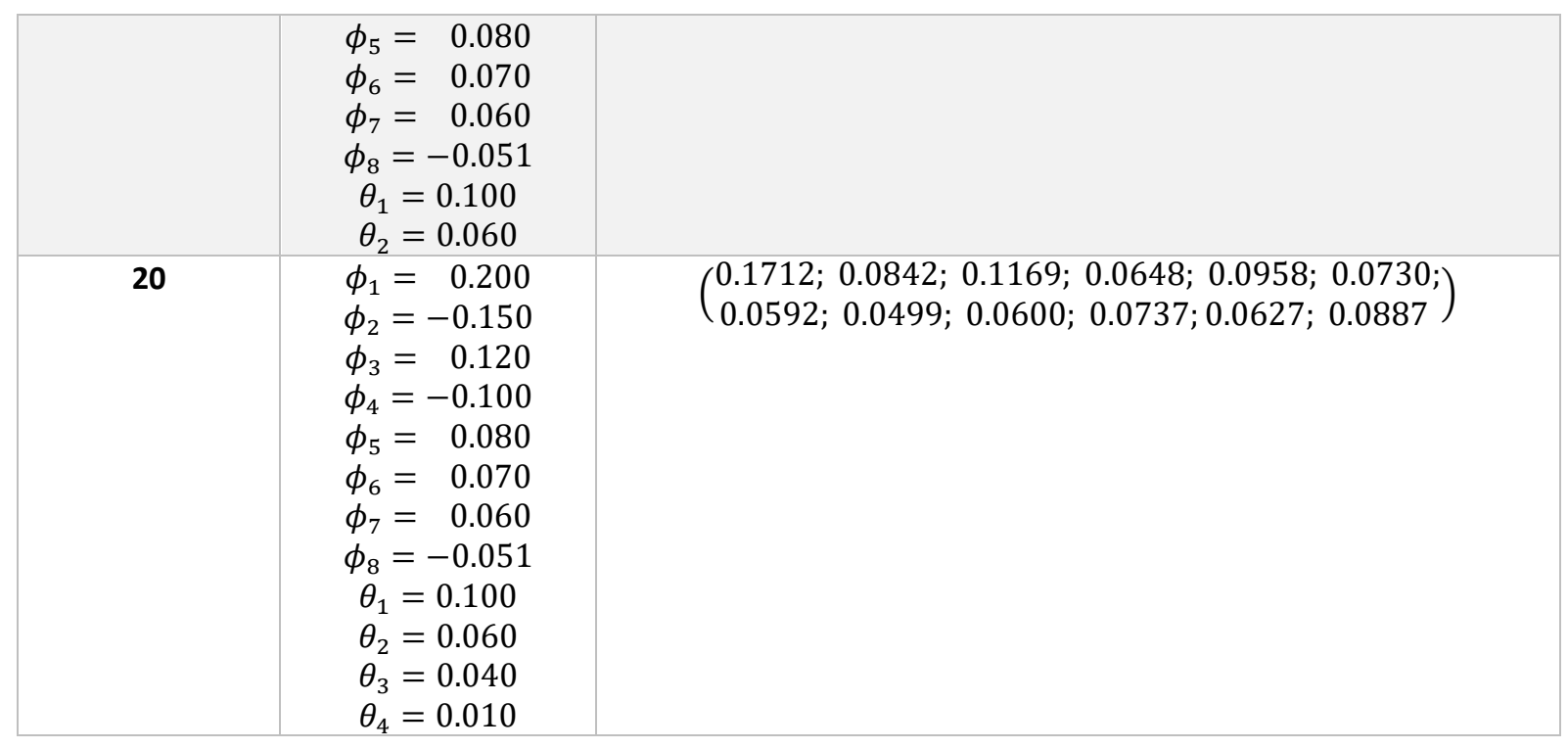

Table 2: Simulation results for SMA and WMA/Newton methods

\begin{tabular}{|c|c|c|c|c|}
\hline \multirow{2}{*}{$\begin{array}{c}\text { Demand } \\
\text { Model }\end{array}$} & \multicolumn{2}{|c|}{ SMA method } & \multicolumn{2}{|c|}{ WMA/Newton method } \\
\hline & $M S E^{D D I}$ & $\tilde{I}_{t}^{D D I}$ & $M S E^{D D I^{*}}$ & $\tilde{I}_{t}^{D D I^{*}}$ \\
\hline $\mathbf{1}$ & 20.3867 & 11.5614 & 19.4392 & 11.4855 \\
\hline 2 & 26.7926 & 14.3894 & 24.6456 & 14.2098 \\
\hline 3 & 36.5808 & 18.7090 & 31.8348 & 18.2922 \\
\hline 4 & 16.2400 & 07.4301 & 15.8909 & 7.4038 \\
\hline 5 & 18.5000 & 07.7789 & 17.9541 & 7.7376 \\
\hline 6 & 20.9400 & 08,1536 & 20.1583 & 8.0942 \\
\hline 7 & 22.3074 & 11.8812 & 21.0996 & 11.7838 \\
\hline 8 & 24.2527 & 12.2041 & 22.7645 & 12.0835 \\
\hline 9 & 33.2078 & 13.6816 & 30.2764 & 13.4401 \\
\hline 10 & 37.4282 & 14.4392 & 33.4410 & 14.1049 \\
\hline 11 & 39.6913 & 14.8415 & 35.1010 & 14.4536 \\
\hline 12 & 42.0563 & 15.2594 & 36.8125 & 14.8132 \\
\hline 13 & 44.6284 & 15.8453 & 38.2068 & 15.2839 \\
\hline 14 & 19.6140 & 10.8756 & 18.5583 & 10.7887 \\
\hline 15 & 24.1279 & 15.9735 & 20.8552 & 15.6680 \\
\hline 16 & 26.5067 & 16.4101 & 22.4153 & 16.0256 \\
\hline 17 & 29.7530 & 17.0342 & 23.9784 & 16.4813 \\
\hline 18 & 12.8447 & 8.4158 & 12.5980 & 8.3972 \\
\hline 19 & 13.8480 & 8.6012 & 13.5038 & 8.5747 \\
\hline 20 & 14.4736 & 8.7396 & 14.0079 & 8.7030 \\
\hline
\end{tabular}


For all simulated models in Tab. 2, the WMA/Newton method proves its efficiency and overweighs the SMA method with regard to the two performance metrics. Therefore, this table shows that DDI performance depends on the weighting vector itself. By the same way, it proves that decision-makers in supply chains can enhance their competitiveness in the market by simply considering the optimal weighting generated by Newton's method rather than considering equitable weighting of the order of $1 / N$. As expected, the enhancement in the two metrics is different as we vary the autoregressive and moving average coefficients of the demand models. For example, for the first model, the yield in terms of mean squared error is equal to $\left|\frac{19.4392-20.3867}{20.3867}\right| \times 100 \approx 4.67 \%$ and the yield in terms of average inventory level is equal to $\left|\frac{11.4855-11.5614}{11.5614}\right| \times 100 \approx 0.65 \%$. This is due to the non-linear relation mentioned before (3c), between the forecast mean squared error, and its effective consequence (here is the average inventory level). It's also clear that the demand time-series structure influences these enhancements: while the yield for the first model is $0.65 \%$ in terms of average inventory level, the yield for the seventieth model is about 3.24\%. These enhancement percentages are not negligible when we know the sizes of inventories in the real world economy, and their respective inventory costs.

\subsection{Sensibility of WMA method with respect to Moving Average parameter}

In this part of simulation, we suppose that both supply chain partners agreed to adopt DDI strategy with a WMA/Newton forecasting methods. Generally, the lead-time value is an unchangeable datum which depends on transportation and logistics systems, and managers do not really have a real power (a very large margin) to variate its value. However, the moving average parameter $N$ depends on the manager's choice about the horizon of considered past observations.

As there is no structured ways to find out the "optimal" moving average parameter $N$ of the WMA method, we study the sensibility of the two performance metrics with respect to $N$. Thus, for illustration, we consider arbitrarily an example of $A R M A(3,2)$ demand process as follows:

$$
D_{t}=10+0.6 D_{t-1}+0.4 D_{t-2}-0.3 D_{t-3}+\xi_{t}+0.1 \xi_{t-1}+0.08 \xi_{t-2}
$$

Fig. 1 presents the simulation's results in terms of mean squared errors and average inventory level improvements in percentage, for a fixed value of lead-time $L$ while $N$ is varying between 8 and 20.

Figure 1: Improvements of mean squared error and average inventory level with regard to moving average parameter $N$ 


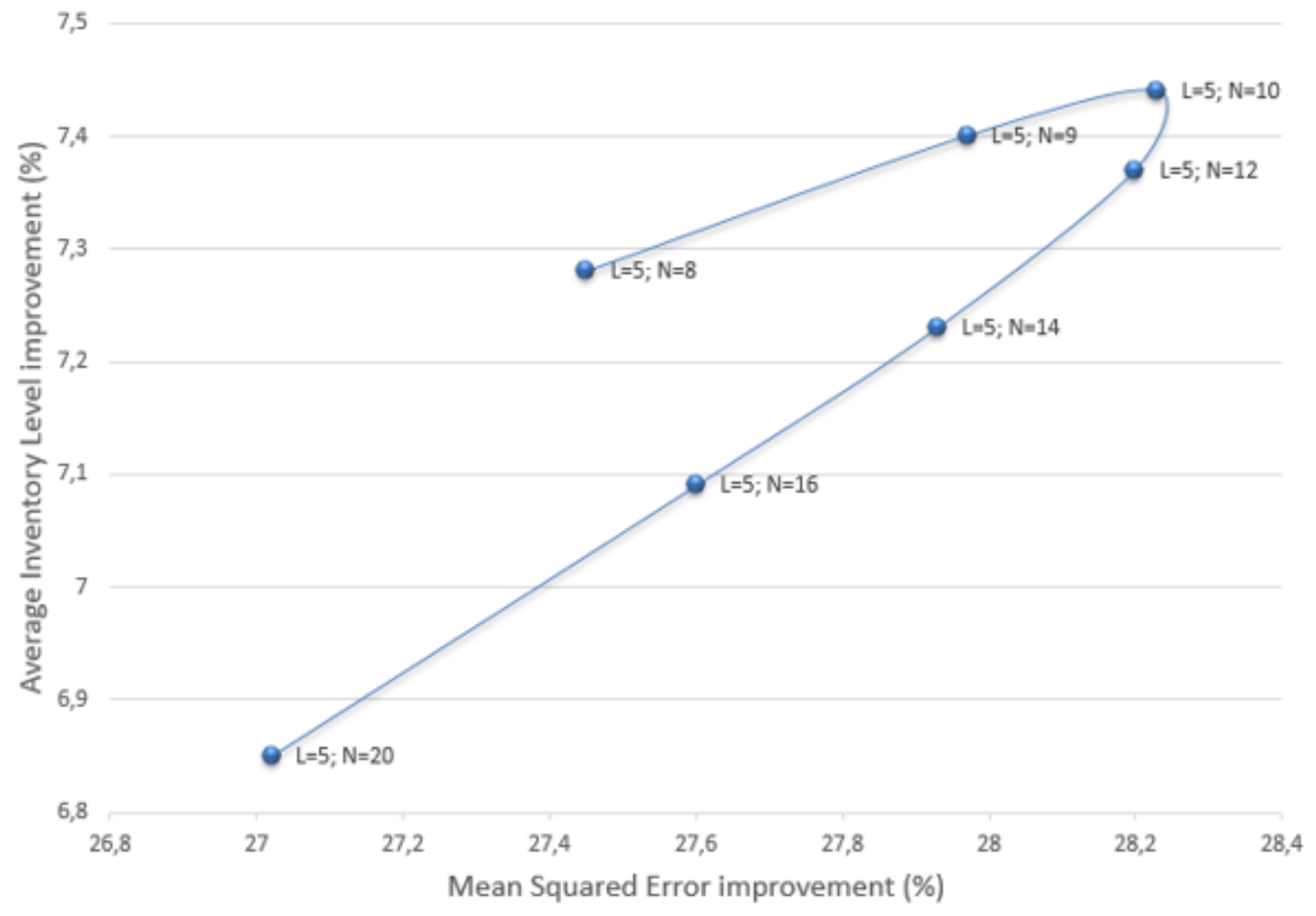

The results obtained in Fig. 1 show that, for $L=5$, the evolution with respect to $N$ is a concave function on the local interval. These results show that this function attains approximatively its local maximal enhancement at $N=10$. This optimum corresponds to 7.44\% in average inventory savings. Otherwise, the enhancement still exists but is not optimal. In practice, the decision-makers can make some simulations by varying the parameter $N$ on a fixed interval and choose the value that locally maximizes this enhancement. Further analytical investigation can reveal global maximal improvements.

\section{Conclusion}

In decentralized supply chains, actors often do not want to share their private information especially when it comes to an explicit sharing of the market demand. This variable is often considered as a variable holding a competitive power. Even in cases where supply chain actors are favorable to information sharing, other issues may still persist.

This paper is a follow-up study to previous works with the purpose of improving existing DDI results through theoretical analysis of inventory models based on some strong assumptions. In a context of DDI coordination strategy, instead of using SMA method, we propose to use of the WMA method combined with the well-known Newton optimization method. 
This work provides an initial attempt to introduce WMA forecasting method, in a decentralized supply chain which actors are favorable to adopt DDI strategy. The propagation of processes with WMA forecast method, is unique. The introduction of Newton optimization method allows the quantification of the weighting of past demand observations according to the purpose of average inventory minimization. The study of improvements according to moving average parameter $N$ showed that supply chains decision-makers are able to estimate approximatively, which historical horizon value is locally optimal. Practitioners can easily change the $N$ value in the forecasts.

It's firstly important for decision-makers to further reduce inventory levels and gathering additional savings. Indeed, the resulting reduction in the upstream actor average inventory level translates into cost savings over time. These savings are the most important engine inciting for DDI adoption. Our work shows through simulation, that savings through WMA/Newton approach, exceed savings through SMA method. We estimate that the Newton's method itself is not expensive in terms of time-implementation. While it is natural to expect a distribution of these savings between the upstream actor and downstream actor, coordination is essential to achieve such improvements.

In this paper, we have considered the enhancement of forecast error and inventory level metrics as they are directly related to inventory costs. One future research direction can be the consideration of bullwhip effect measure.

This approach does not require further assumptions than those required by DDI with SMA method, namely the knowledge of demand process coefficients along the supply chain. Besides, it's also essential to considerate costs of such coordination. These costs are primarily related to the implementation of the method itself (if another forecast method was adopted by the downstream actor), systems compatibility and Newton weighting information sharing.

WMA/Newton forecasting approach for DDI strategy can be setup through different managerial contracts between the upstream actor and the downstream actor. The literature is abundant of such contracts. For example, the upstream actor may propose contracts to the downstream actor based on principal agent (Müller \& Turner, 2005), or they can negotiate through proposal generation (Dudek \& Stadtler, 2005). Buyback (Chen \& Bell, 2011) or pricediscounts (Jain et al., 2011) contracts may also be proposed.

We conclude our paper with natural lines for future researches. First, DDI strategy can still be evaluated through other forecasting methods. Second, it would be interesting to adopt the bullwhip effect as the objective function of WMA/Newton methods. Another direction is the consideration of multi-objective optimization for parallel improvement of supply chain performance metrics.

\section{Acknowledgment}

This paper is an output of the science project PERFAD, which is co-financed by the European Union with the European Development Fund and the Regional Council of Normandy

\section{References}

Ali, M. M., Babai, M. Z., Boylan, J. E., \& Syntetos, A. A. (2017). Supply chain forecasting when information is not shared. European Journal of Operational Research, 260(3), 984-994. 
Ali, M. M., \& Boylan, J. E. (2012). On the effect of non-optimal forecasting methods on supply chain downstream demand. IMA Journal of Management Mathematics, 23(1), 81-98.

Ali, M. M., \& Boylan, J. E. (2011). Feasibility principles for Downstream Demand Inference in supply chains. Journal of the Operational Research Society, 474-482.

Ali, M. M., Boylan, J. E., \& Syntetos, A. A. (2012). Forecast errors and inventory performance under forecast information sharing. International Journal of Forecasting, 28(4), 830-841.

Cachon, G. P., \& Fisher, M. (2000). Supply chain inventory management and the value of shared information. Management science, 46(8), 1032-1048.

Chen, J., \& Bell, P. (2011). The impact of customer returns on decisions in a newsvendor problem with and without buyback policies. International Transactions in Operational Research, 18(4), 473-491.

Chen, B., Chen, X., \& Kanzow, C. (2000). A penalized Fischer-Burmeister NCPfunction. Mathematical Programming, 88(1), 211-216.

Dudek, G., \& Stadtler, H. (2005). Negotiation-based collaborative planning between supply chains partners. European Journal of Operational Research, 163(3), 668-687.

Fawcett, S. E., Osterhaus, P., Magnan, G. M., Brau, J. C., \& McCarter, M. W. (2007). Information sharing and supply chain performance: the role of connectivity and willingness. Supply Chain Management: An International Journal, 12(5), 358-368.

Forslund, H., \& Jonsson, P. (2007). The impact of forecast information quality on supply chain performance. International Journal of Operations \& Production Management, 27(1), 90-107.

Gordon, G., \& Tibshirani, R. (2012). Karush-kuhn-tucker conditions. Optimization, 10(725/36), 725.

Jain, A., Seshadri, S., \& Sohoni, M. (2011). Differential pricing for information sharing under competition. Production and Operations Management, 20(2), 235-252.

Klein, R., Rai, A., \& Straub, D. W. (2007). Competitive and cooperative positioning in supply chain logistics relationships. Decision Sciences, 38(4), 611-646.

Lee, H. L., \& Whang, S. (2000). Information sharing in a supply chain. International Journal of Manufacturing Technology and Management, 1(1), 79-93.

Lee, H. L., So, K. C., \& Tang, C. S. (2000). The value of information sharing in a two-level supply chain. Management science, 46(5), 626-643.

Mendelson, H. (2000). Organizational architecture and success in the information technology industry. Management science, 46(4), 513-529.

Müller, R., \& Turner, J. R. (2005). The impact of principal-agent relationship and contract type on communication between project owner and manager. International Journal of Project Management, 23(5), 398-403. 


\section{International Conference on}

\section{MANAGEMENT, ECONOMICS \& FINANCE}

Qi, L., \& Sun, D. (1999). A survey of some nonsmooth equations and smoothing Newton methods. In Progress in optimization (pp. 121-146). Springer, Boston, MA.

Sahin, F., \& Robinson, E. P. (2005). Information sharing and coordination in make-to-order supply chains. Journal of operations management, 23(6), 579-598.

Shumway, R. H., \& Stoffer, D. S. (2011). ARIMA models. In Time Series Analysis and Its Applications (pp. 83-171). Springer, New York.

Silver, E. A., Pyke, D. F., \& Peterson, R. (1998). Inventory management and production planning and scheduling (Vol. 3, p. 30). New York, Wiley.

Sorensen, D. C. (1985). Analysis of pairwise pivoting in Gaussian elimination. IEEE Transactions on Computers, (3), 274-278.

Tliche, Y., Taghipour, A., \& Canel-Depitre, B. (2019). Downstream Demand Inference in decentralized supply chains. European Journal of Operational Research, 274(1), 65-77.

Yu, Z., Yan, H., \& Edwin Cheng, T. C. (2001). Benefits of information sharing with supply chain partnerships. Industrial management \& Data systems, 101(3), 1 